\title{
Influence of Gas-Liquid Interface on Temperature Wave of Pulsating Heat Pipe
}

\author{
Ying Zhang, Zhiqiang Wang, Peisheng Li $(\mathbb{D}$, Min Zhou, Boheng Dong, and Yanni Pan \\ College of Mechanical and Electrical Engineering, Nanchang University, Nanchang 330031, China \\ Correspondence should be addressed to Peisheng Li; ncudns1995z@163.com
}

Received 31 August 2017; Revised 22 December 2017; Accepted 11 January 2018; Published 11 March 2018

Academic Editor: Xinhai Xu

Copyright (C) 2018 Ying Zhang et al. This is an open access article distributed under the Creative Commons Attribution License, which permits unrestricted use, distribution, and reproduction in any medium, provided the original work is properly cited.

\begin{abstract}
The influence of the interface on the amplitude and phase of the temperature wave and the relationship between the attenuation of the temperature wave and the gas-liquid two-phase physical parameters are studied during the operation of the pulsating heat pipe. The numerical simulation shows that the existence of the phase interface changes the direction of the temperature gradient during the propagation of the temperature wave, which increases the additional "thermal resistance." The relative size of the gas-liquid two-phase thermal conductivity affects the propagation direction of heat flow at phase interface directly. The blockage of the gas plug causes hysteresis in the phase of the temperature wave, the relative size of the gas-liquid two-phase temperature coefficient will gradually increase the phase of the temperature wave, and the time when the heat flow reaches the peak value is also advanced. The attenuation of the temperature wave is almost irrelevant to the absolute value of the density, heat capacity, and thermal conductivity of the gas-liquid two phases, and the ratio of the thermal conductivity of the gas-liquid two phases is related. When the temperature of the heat pipe was changed, the difference of heat storage ability between gas and liquid will lead to the phenomenon of heat reflux and becomes more pronounced with the increases of the temperature wave.
\end{abstract}

\section{Introduction}

Pulsating heat pipe (PHP) is an efficient and energy-saving heat exchanger element $[1,2]$. Solar collectors are the main equipment of solar energy equipment, and the combination with PHP can improve the heat transfer efficiency [3]. When using a PHP as a thermal management tool [4], the temperature of the heat management object changes frequently $[5,6]$, whose temperature and power vary constantly as the working load changes. Whether the PHP can respond quickly to such a temperature change is extremely important for heat management. Therefore, as the core component of solar collector, solar heat pipe plays an important role in collecting heat and safety of the collector [7]. The flow characteristics of the working fluid in the pulsating heat pipe determine the operating mechanism and heat transfer characteristics of the PHP, so the study of the flow law is the basis of the theoretical analysis and the establishment of the mathematical model [8]. The pulsating heat pipe undergoes complex gas-liquid flow patterns and transitions such as bubble flow, plunger flow, annular/semiannular flow during the start-up phase as well as the alternating cycles between the annular flow of the initial circulation stage and plunger flow in the tube [9]. Jiansheng et al. [10] use the numerical simulation method to demonstrate that different flow patterns can reduce the heat transfer resistance and enhance the heat transfer capacity in the PHP starting and running. In order to evaluate the heat transfer performance of the PHP accurately, it is necessary to examine the radial heat transfer, evaporation heat transfer, and condensation heat transfer of the working medium and the wall [11]. Kim et al. [12] studied the effect of the temperature pulsation at the evaporating section and the condensate section on the performance of heat pipe, it was found that the sensible heat transfer in the heat transfer mode of the PHP was dominant, and the oscillation frequency of the liquid slug decreases with the increase of the frequency and amplitude of the wall temperature with the periodic pulsation, and the heat transfer performance of the PHP depends on the intensity of the pulsation [13]. Zhang et al. [14] studied the effects of variable bend, number of the liquid slug and the air slug, and the amplitude and frequency of the oscillating flow in the PHP; 
the result showed that the influence of the liquid slug and the air slug was small when the number of bends was less than 5 , and the amplitude and frequency of the oscillating will increase with the increase of the filling rate. In addition, by studying the movement of gas slug and liquid slug in $\mathrm{U}$ tube, it is found that the amplitude and frequency of pulsation of gas slug and liquid slug increase with the increase of temperature difference between evaporation section and condensation section [15].

However, scholars mainly focus on the overall heat dissipation of PHP, and the research on the flow characteristics of the working fluid in PHP is less. As measuring and controlling the temperature in the experiment are difficult, and many factors affect the flow pattern, it is difficult to obtain a stable flow pattern in PHP. Therefore, this paper constructs a phase interface containing a gas slug in a rectangular area and simulates the related phenomena under the condition of low power start and power change. Since any continuous periodic fluctuation curve can be superimposed with multiple sine or cosine functions $[16,17]$, a sinusoidal temperature is added at one end of the rectangle. The influence of temperature fluctuation on the operation of PHP is analyzed by studying the variation of heat flow and heat flux as well as the amplitude and phase of temperature wave.

\section{Computational Model and Numerical Method}

2.1. Computational Model. According to the working state of $\mathrm{PHP}$, a computational domain of gas-liquid interface is constructed. Since the time of the movement of the air slug is much shorter than the time of the temperature changes in the steady state of the PHP, it is assumed that the air slug remains stationary during the temperature propagation in order to facilitate the study. As shown in Figure 1, the area of the computational domain is $2 \mathrm{~mm} \times 1 \mathrm{~mm}$. The upper boundary and lower boundary are solid boundary, and the left boundary and the right boundary are the pressure boundary. The coordinates of points 1, 2, and 3 are $x=0.4 \mathrm{~mm}, y=-0.5 \mathrm{~mm} ; x=0.743 \mathrm{~mm}, y=-0.5 \mathrm{~mm}$; and $x=1.6 \mathrm{~mm}, y=0.5 \mathrm{~mm}$ (located in front of the gas slug, inside the gas slug, and behind the gas slug).

2.2. Numerical Methods. In this paper, the VOF model is adopted, and the control equation is shown as follows:

(1) Continuity equation:

$$
\frac{\partial \rho}{\partial t}+\frac{\partial}{\partial x_{i}}\left(\rho u_{i}\right)=0
$$

(2) Momentum conservation equation:

$$
\frac{\partial}{\partial t}\left(\rho u_{i}\right)+\frac{\partial}{\partial x_{i}}\left(\rho u_{i} u_{j}\right)=-\frac{\partial p}{\partial x_{i}}+\frac{\partial \omega_{i j}}{\partial x_{j}}+\rho g_{i}+S_{N},
$$

wherein $P$ is the pressure, $g_{i}$ is the volume force in the $i$ direction, and $S_{N}$ is the source term which is a continuum surface force term.

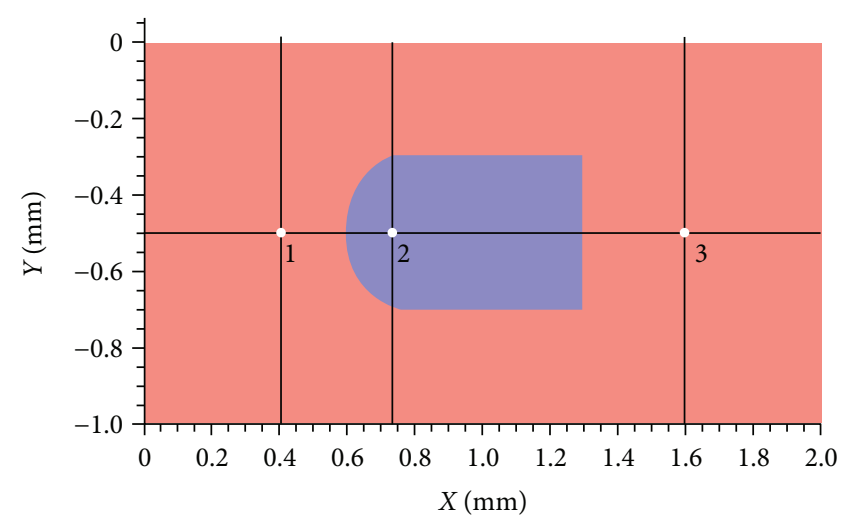

FIGURE 1: Computational model (the light area is the liquid phase area, and the dark area is the gas phase area).

The stress tensor is shown below:

$$
\omega_{i j}=u\left(\frac{\partial u_{i}}{\partial x_{i}}+\frac{\partial u_{j}}{\partial x_{j}}\right)-\frac{2}{3} \frac{\partial u_{l}}{\partial x_{l}} \delta
$$

(3) The energy equation:

$$
\frac{\partial(\rho T)}{\partial t}+\operatorname{div}(\rho u T)=\operatorname{div}\left(\frac{\lambda}{c} \operatorname{grad} T\right)+S_{T} .
$$

Taken as the microelement at the interface, which is assumed that its physical parameters are equal, the equation of heat conduction can be expressed by the following formula [18]:

$$
\frac{\partial T}{\partial t}=\frac{\lambda}{\rho c}\left(\frac{\partial^{2} T}{\partial x^{2}}+\frac{\partial^{2} T}{\partial y^{2}}\right)
$$

wherein $T$ is the temperature, $t$ is the time, $\lambda$ is the thermal conductivity, $c$ represents the heat capacity at constant pressure, and $S_{T}$ is viscous dissipation term.

In this paper, the liquid phase is the main phase, and the volume fraction of liquid phase is obtained by the following equation:

$$
\varphi_{L}=1-\varphi_{G},
$$

wherein $\varphi_{L}$ is the volume fraction of liquid phase; $\varphi_{G}$ is the volume fraction of gas phase. And $\varphi_{G}$ is obtained by the following formula:

$$
\frac{\partial}{\partial t}\left(\rho_{g} \varphi_{g}\right)+\Delta\left(\rho_{g} \varphi_{g} u_{g}\right)=0 .
$$

The computational model shown in Figure 1 is divided into unstructured meshes. After completing the grid independence check, the total number of grids adopted was 21980. In this paper, Ansys16.0 Fluent is used to solve the model control equation. Initially, the left boundary temperature is $300 \mathrm{~K}$, the right boundary using the first type of boundary conditions is $300 \mathrm{~K}$ constantly, and the upper and lower boundaries are set to be adiabatic and solid boundary. 
The UDF file on the left border was imported with a period of $5100 \mathrm{~s}$ and a sine temperature waveform with an amplitude of $100 \mathrm{~K}$. The simulation parameters used in this paper are given in Table 1, and assume that these parameters do not change with temperature. Because the scale of the model is very small, the internal gas-liquid two-phase flow belongs to the typical confined laminar flow; therefore, the basic laminar flow model is used in the simulation, and the pressure field and velocity field are coupled in the solution. The PISO method is introduced, which includes the near correction and the skewness correction. In this paper, the residual convergence criterion of each parameter in the flow field is $10^{-5}$.

\section{Results Analysis}

3.1. The Influence of Phase Interface on Heat Flow. The existence of phase interface changes the direction of propagation of temperature. Initially, the temperature gradient in the $Y$ direction is almost zero, and the isotherm is approximately parallel to the $y$-axis. When the temperature propagates near the phase interface, as shown in Figures 2(a)-2(c), there is no temperature change in the flow field without the interface, a temperature gradient in the $Y$ direction is generated near the arc phase interface, and the central normal of phase interface is symmetrical axis and is symmetrically distributed on both sides. In the area near the center of the curved interface, the temperature gradient is not obvious due to the fact that the upper and lower heat flows are almost simultaneous. In contrast to Figures 2(b) and 2(c), it can be found that the direction of the temperature gradient is related to the thermal conductivity of the gas-liquid two phases. When the thermal conductivity of the liquid phase is greater than that of the gas phase, the direction of the temperature gradient is outward. When the thermal conductivity of the liquid phase is less than that of the gas phase, the direction of the temperature gradient is inward.

The propagation of thermal waves is similar to that of electromagnetic waves, when passing through propagation medium of different density, both the reflection and the refraction occur, the ratios of reflection and refraction are determined by the thermal conductivity of the two phases. Similar to the Kirchhoff law, heat flow always flows in the direction of less thermal resistance. When the heat flow passes through the phase interface, its orientation is related to the relative thermal conductivity of the internal and external materials at the interphase interface and always tends to the material with large thermal conductivity. Figures 3(a) and 3(b) are corresponding to physical parameters of Figures 2(b) and 2(c), respectively. In combination with Figures 2 and 3, it is found that when the thermal conductivity of the gas is less than that of the liquid phase, the temperature gradient near the interface is outward and the heat flow passes through the gas slug inward. When the thermal conductivity of the gas is larger than that of the liquid phase, the temperature gradient near the interface is inward, and the heat flow passes around the gas slug outward.

\subsection{Influence of Phase Interface on Phase and Amplitude of Temperature Wave}

3.2.1. Influence of Phase Interface on Temperature Wave Phase. The attenuation degree of temperature wave is different in the different area of gas-liquid, and the change of the temperature wave phase may advance or lag. When the period of temperature wave is large, the change of the temperature wave is not obvious in the informal state, whether it changes its physical parameter or position coordinate, and the phase of all the positions in the computational domain is almost the same. When the period of the temperature wave is small, the apparent irregular state can be seen, and the phases in different positions of the computational domain are obviously different.

For the sake of study, the abscissa of the case b in Figure 4(a) is reduced by a factor of 15 , the periods of case $\mathrm{b}$ and case e are $100 \mathrm{~s}$ and $5 \mathrm{~s}$, respectively. In Figure 4, A-I represents the case A of position I. As shown in case e, by comparing curves e- 1 and e- 2 , it can be found that the phase lag of the temperature wave in the gas phase is about $20 \%$ along the $x$-axis, the time after reaching the highest temperature is delayed, and the amplitude of the temperature wave is also reduced by $30 \%$.

Figure 4(b) shows the change of the phase of the temperature wave with or without phase interface. Case e and case 1 are selected, $y$ is $-0.5 \mathrm{~mm}$, and the values of $x$ were different (as shown in the model 1, 2, and 3 points). It can be seen from the figure that the presence of the phase interface changes the phase of the temperature wave. Compared with several sets of curves, it is found that the phase of the curve without phase interface is ahead of the temperature wave curve with phase interface before the temperature wave propagates to the phase interface. With the increase of the $x$-coordinate, the temperature wave with phase interface will gradually advance the temperature wave without phase interface. This is mainly because the thermal diffusion coefficient of the liquid phase is substantially the same in the front of the gas slug, but the obstruction of the gas slug causes the heat flow in the local area close to the phase interface to be reduced. Therefore, the temperature of this region peaks faster than that of the region with the phase interface. Behind the phase interface, the thermal diffusivity of gas is greater than the liquid, so the change of the temperature of the gas phase is faster than that of the liquid phase, resulting in the temperature of the region with phase interface peaks faster than that of the region without the phase interface. The phase advance and hysteresis have great influence on the propagation of temperature. This is because the process of starting and running of the whole heat pipe has always been the alternate change of gas-liquid phase. The existence of phase interface is bound to affect the speed of temperature propagation.

\subsubsection{Influence of Phase Interface on the Amplitude of} Temperature Wave. It is significant to understand the change of the temperature of the gas-liquid two-phase working fluid in the heat pipe by studying the attenuation of the temperature wave when the temperature of the outer wall of the heat pipe changes. Figure 5 shows the attenuation curve of the 


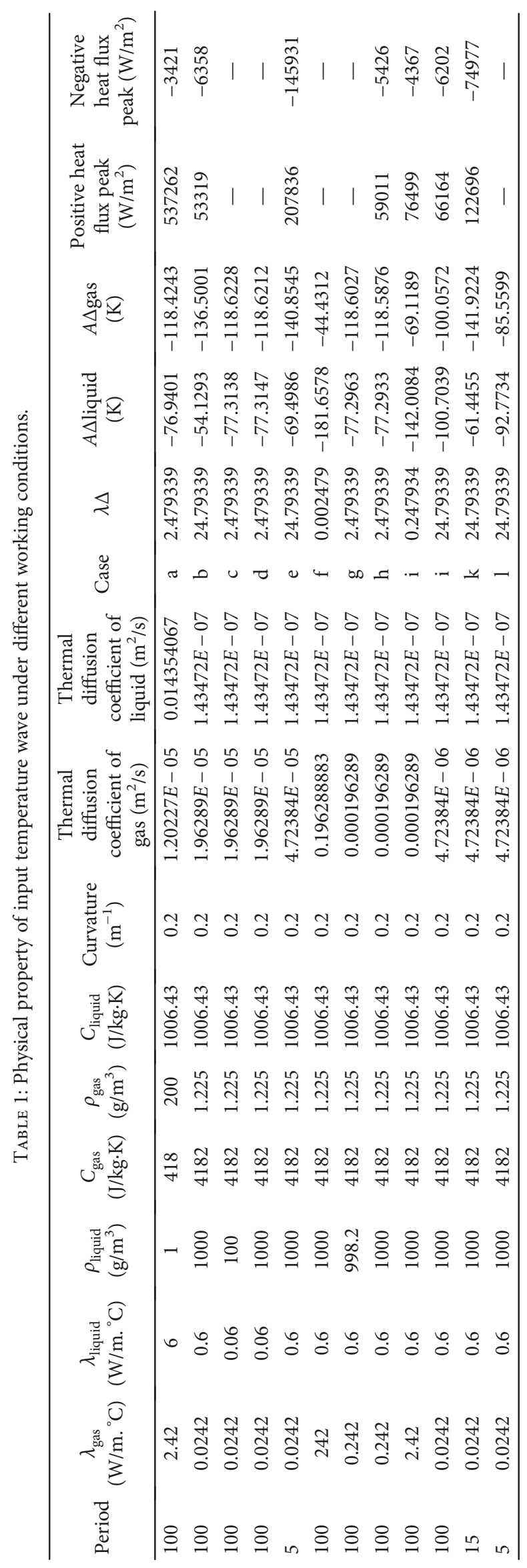




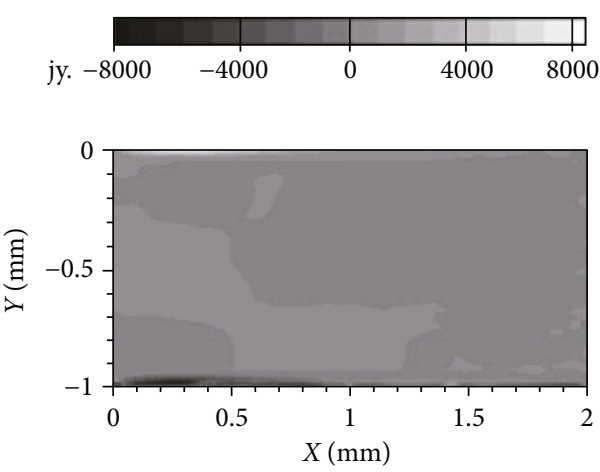

(a) Nonphase interface
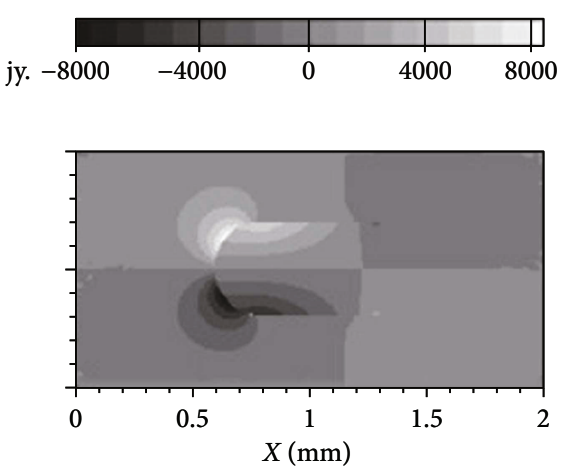

(b) $\lambda_{l}>\lambda_{g}$
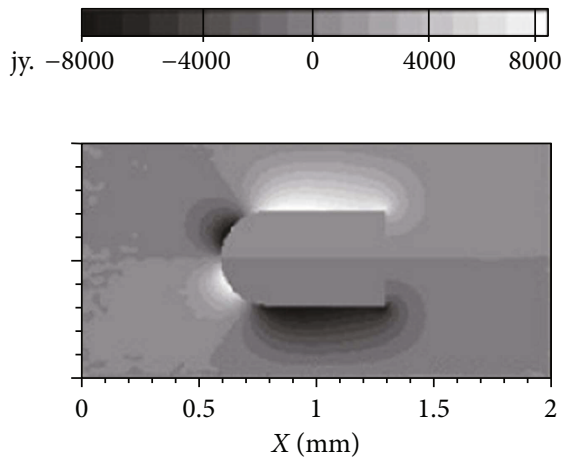

(c) $\lambda_{l}<\lambda_{g}$

FIgURE 2: Temperature gradient diagram of $Y$ direction with or without phase interface.

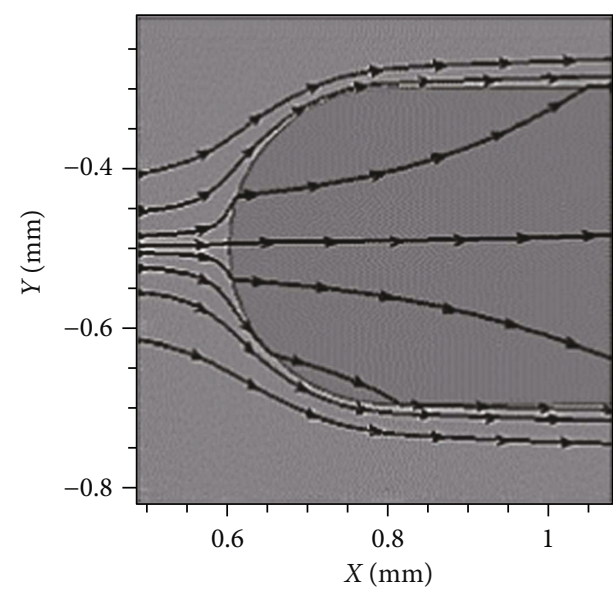

(a) $\lambda_{l}>\lambda_{g}$

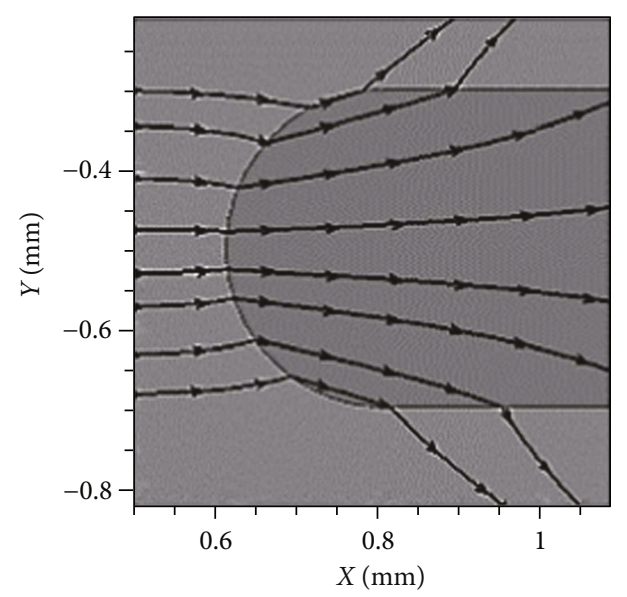

(b) $\lambda_{l}<\lambda_{g}$

FIGURE 3: Vector diagram of heat flow through a phase interface (the light-colored area is the liquid phase, and the dark area is the gas phase).

amplitude of the temperature wave on the axis of the air plug in different working conditions. It can be found that the curvature of the curve will change obviously near the position of the phase interface, and this position is located in the front of the interface. Before and after the curvature changes, the temperature wave in each condition attenuates by approximately equal attenuation coefficient. In the gas phase section, since the heat flow is no longer propagating in the original direction, the attenuation coefficient of the gas phase section is significantly smaller than the temperature attenuation coefficient of the liquid phase section, regardless of whether the thermal conductivity of the gas phase is larger or smaller than that of the liquid phase. At the same time, it can be seen from Figure 5 that the attenuation coefficient of case $b$ is the smallest and the attenuation coefficient of the case $f$ is the largest. However, the attenuation curves of a, c, d, and $\mathrm{k}$ are almost coincident. Combined with Table 1, it can be concluded that the temperature attenuation coefficient has nothing to do with the density and the heat capacity of the material and is also not significantly related to the value of 


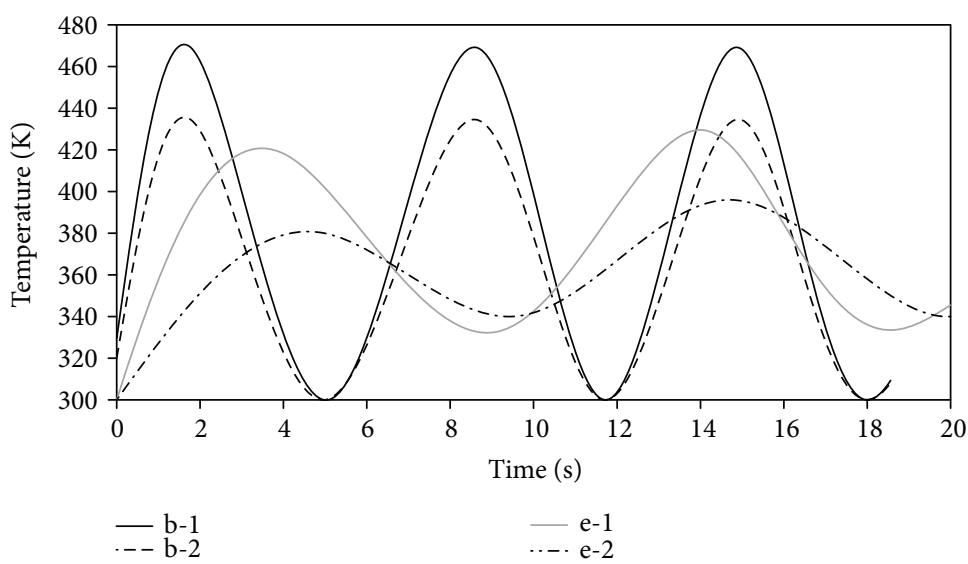

(a) Waveform of different periods and positions

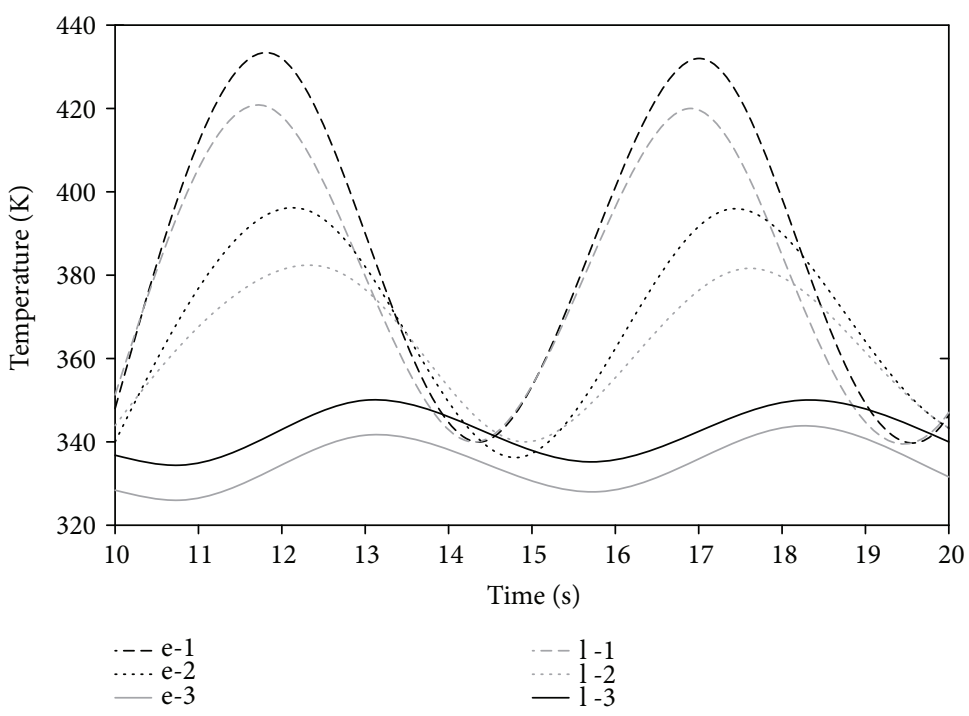

(b) The variation of temperature wave with time in different positions of the same period

FIgURE 4: Curves of waveform versus time.

the thermal conductivity of the material, considering that the difference in thermal conductivity could lead to the direction of the heat flow change at the phase interface. There defines a new variable $\lambda_{\Delta}=\lambda_{l} / \lambda_{g}$ which is used to investigate the attenuation of a temperature wave in a heat pipe. Table 1 shows the $\lambda_{\Delta}$ and $A_{\Delta}$ for some of the conditions. As can be seen from the table, $A_{\Delta}$ and $\lambda_{\Delta}$ are significantly related. Before the attenuation coefficient changes, the correlation coefficient of the two variables can be calculated by the correlation formula:

$$
\rho_{\lambda \Delta \cdot A \Delta}=\frac{\operatorname{COV}\left(\lambda_{\Delta}, A_{\Delta}\right)}{\sqrt{D\left(\lambda_{\Delta}\right)} \sqrt{D\left(\lambda_{\Delta}\right)}},
$$

wherein $\operatorname{COV}\left(\lambda_{\Delta}, A_{\Delta}\right)$ is the covariance of $\lambda_{\Delta}$ and $A_{\Delta}$ and $\sqrt{D\left(\lambda_{\Delta}\right)}$ and $\sqrt{D\left(A_{\Delta}\right)}$ are the mean square deviations of $\lambda_{\Delta}$ and $A_{\Delta}$, respectively. Substituting the experimental data can obtain that the correlations in the liquid phase area are 0.6414 and 0.673 , respectively, which is strongly correlated.
After the curvature changes obviously, the correlation coefficient becomes -0.6714 , but it remains strong correlation. As the experimental data increases, the correlation coefficient will be closer to 1 . Then, it is found that $A_{\Delta}$ and $\lambda_{\Delta}$ are approximately logarithmic function by fitting the two sets of data, and the confidence of the fitting is $97 \%$. In the liquid phase section, the base number of logarithm is less than 1 , and in the gas phase section, the logarithmic base is greater than 1, the relationship between the specific function as follows:

$$
\begin{aligned}
& A_{\Delta}=4.989 \times \log _{3 / 2}(x)+97.97 \text { (gas), } \\
& A_{\Delta}=5.625 \times \log _{2 / 3}(x)+99.68 \text { (liquid). }
\end{aligned}
$$

Figure 6 is a curve of the attenuation coefficient of the gas phase and the liquid region with the coefficient of thermal conductivity. In the liquid region, the attenuation coefficient $A_{\Delta}$ decreases with the increase of liquid-gas ratio $\lambda_{\Delta}$, and the rate of change is gradually reduced, finally stabilized near a 


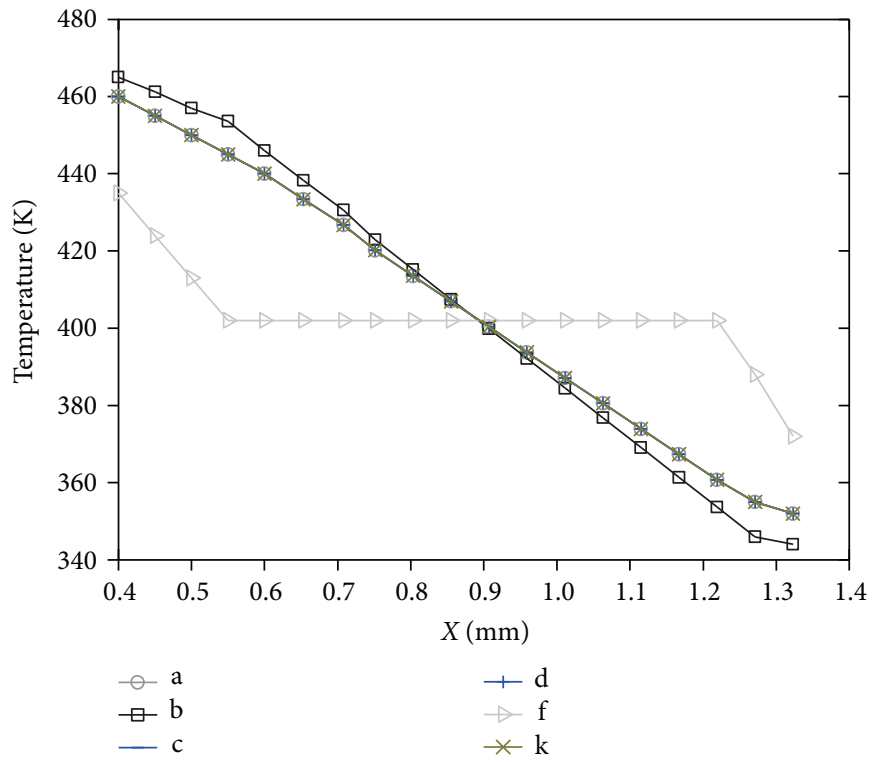

Figure 5: Attenuation curve of temperature wave on the central axis of gas slug under different working conditions (the two vertical dashed lines whose location is $0.6 \mathrm{~mm}$ and $1.3 \mathrm{~mm}$, resp., indicate the phase interface positions).

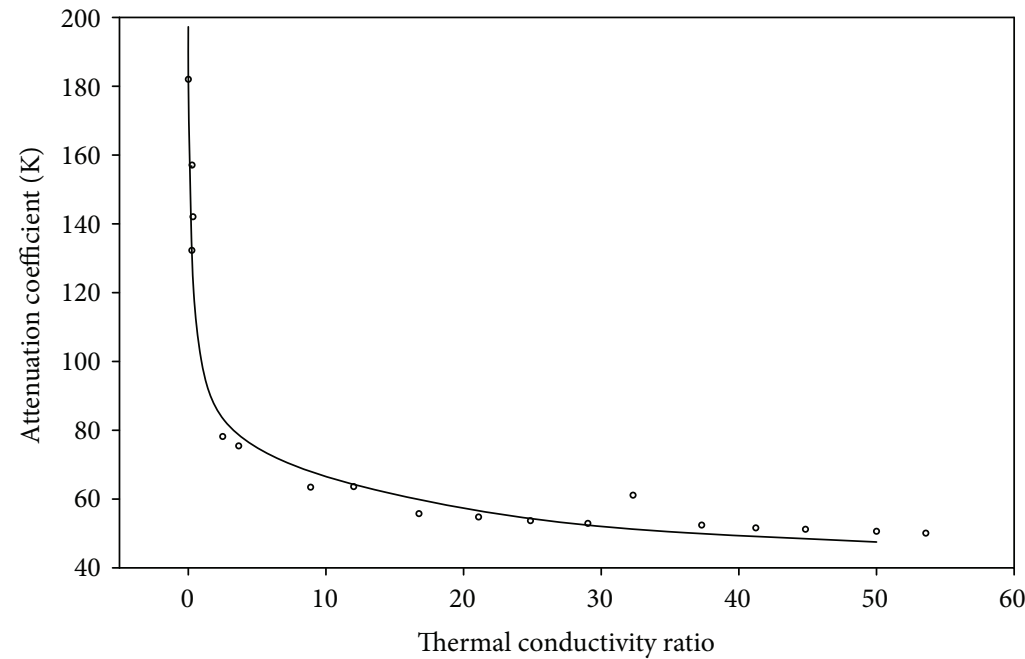

(a) Liquid region

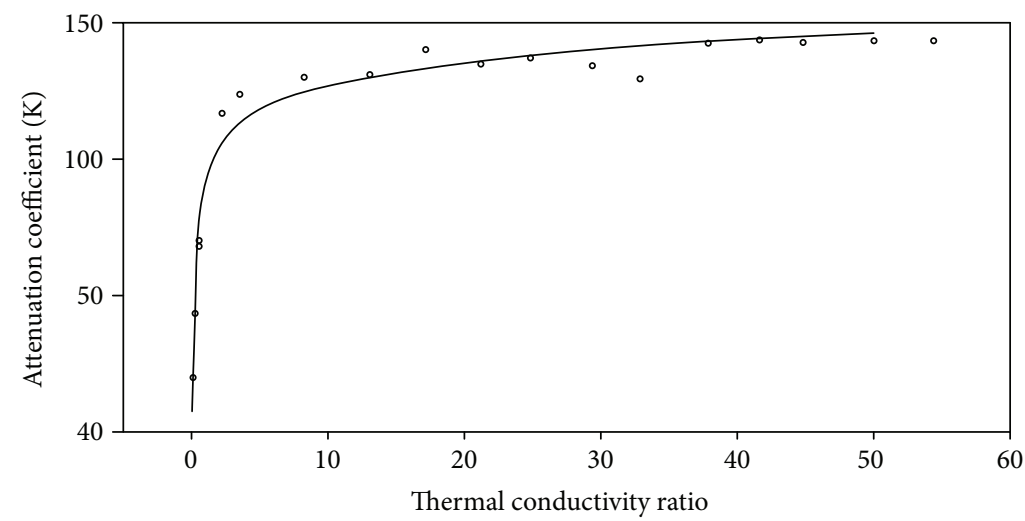

(b) Gas region

FIGURE 6: Curve of attenuation coefficient versus thermal conductivity ratio. 


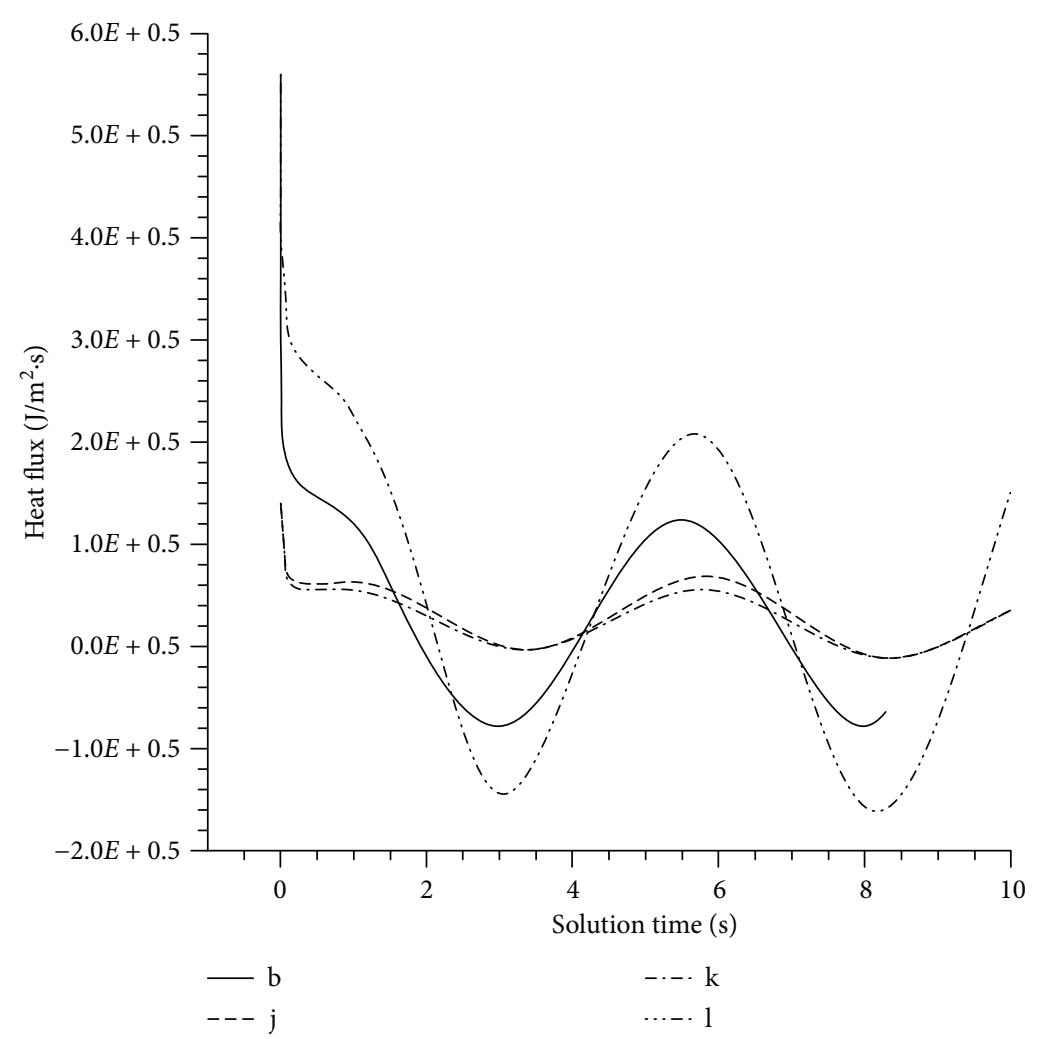

Figure 7: The variation of heat flux with time under different frequencies.

certain value, which is stable near $52 \mathrm{~K}$ in this simulation. However, in the gas region, the attenuation coefficient increases with the increase of $\lambda_{\Delta}$. Similar to the liquid section, the $A_{\Delta}$ eventually stabilized near $142 \mathrm{~K}$.

3.2.3. Influence of Phase Interface on Heat Flux Density. The heat flux is an important indicator of the working state of the heat pipe, and the effect of the phase interface on the heat flux is directly related to the normal and efficient operation of the PHP. Near the left wall, the heat flux is almost equal; therefore, a point near the centerline of the left wall is used to characterize the heat flow in the whole flow field. Figure 7 shows the variation of the heat flux with time under different frequency conditions. Because there mainly research the influence of negative heat flux and in order to make a more intuitive comparison, the abscissa of the period $100 \mathrm{~s}$ and $15 \mathrm{~s}$ is reduced by 10 times and 3 times, respectively. It can be found that due to the delay of the internal temperature change, the heat flow in the opposite direction will occur when the wall temperature drops, which is detrimental to the work of the PHP. By comparing case b and case $\mathrm{j}$, it can be found that the presence of phase interface makes this situation more serious and will also decrease the heat flow peak in the positive direction. By comparing the temperature and heat flux curves at different frequencies, the higher the frequency is, the higher the peak values of heat flow in the positive and negative directions are. There defines a parameter $\Phi$ to measure the intensity of the opposite direction heat flux density. And the greater the absolute value of $\Phi$ is, the greater the intensity of the opposite direction heat flux density is. $\Phi$ is defined as follows:

$$
\Phi=\frac{q_{2}}{q_{1}-q_{2}}
$$

wherein $q_{1}$ is the heat flux density of positive direction and $q_{2}$ is the heat flux density of opposite direction. Under the same conditions of other physical parameters, when the periods are $5 \mathrm{~s}, 15 \mathrm{~s}$, and $100 \mathrm{~s}$, the values of $\Phi$ are $-2.35,-1.571$, and -0.13539 , respectively. It can be found that the frequency of temperature change is high, which is unfavorable to heat pipe heat transfer, that is in line with Kim's research [5]. In the process of starting the heat pipe, the appearance of the two phase is inevitable, so it is necessary to suppress the intensity of the heat flux in the opposite direction.

\section{Conclusions}

In this paper, the influence of the phase boundary in a pulsating heat pipe on the characteristics of temperature wave is simulated and the following conclusions are obtained:

(1) The phase interface changes the propagation direction of the temperature gradient. The propagation direction of heat flux is also changed due to the different thermal conductivity of the gas-liquid two phases. When the thermal conductivity of gas phase is greater than that of liquid phase, the temperature gradient is 
inward at the phase interface, and the heat flow goes outward to bypass the slug. When the thermal conductivity of the gas phase is smaller than the liquid phase, the opposite is true.

(2) Because of the blocking effect of the gas slug, the temperature wave causes phase advance or hysteresis near the phase interface. It can be found that the phase lag of the temperature wave in the gas phase is about $20 \%$ along the $x$-axis.

(3) In the liquid region, the $A_{\Delta}$ decreases with the decrease of the $\lambda_{\Delta}$, and the rate of change is gradually decreased, the $A_{\Delta}$ tends to stabilize near $52 \mathrm{~K}$. But in the gas phase, it is just the opposite, and the $A_{\Delta}$ tends to stabilize near $142 \mathrm{~K}$ eventually.

(4) Because of the delay of the temperature response in the PHP, the phenomenon of thermal reflux is easy to occur when the temperature changes suddenly. In the process of the operation of the PHP, the phase interface will aggravate this phenomenon. And with the increase of the frequency of temperature change, the greater the heat flux in the negative direction is, the worse the heat transfer effect becomes.

\section{Conflicts of Interest}

The authors declare that there is no conflict of interest regarding the publication of this paper.

\section{Authors' Contributions}

Professor Ying Zhang is the first author.

\section{Acknowledgments}

This paper is supported by the National Natural Science Foundation of China (11562011).

\section{References}

[1] D. Haiyan and H. Guo, "Application of heat pipe in solar water heater," Chemical Progress, vol. 3, pp. 390-394, 2008.

[2] Z. Deng, Y. Zheng, X. Liu, B. Zhu, and Y. Chen, "Experimental study on thermal performance of an anti-gravity pulsating heat pipe and its application on heat recovery utilization," Applied Thermal Engineering, vol. 125, pp. 1368-1378, 2017.

[3] C. Chuanbao, "Oscillating heat pipe research and its application in solar collectors," North China Electric Power University, 2009.

[4] J. L. Xu, Y. X. Li, and T. N. Wong, "High speed flow visualization of a closed loop pulsating heat pipe," International Journal of Heat and Mass Transfer, vol. 48, no. 16, pp. 3338-3351, 2005.

[5] K. S. Kim, M. H. Won, J. W. Kim, and B. J. Back, "Heat pipe cooling technology for desktop PC CPU," Applied Thermal Engineering, vol. 23, no. 9, pp. 1137-1144, 2003.

[6] K. Gi, S. Maezawa, Y. Kojima, and N. Yamazaki, "CPU cooling of notebook PC by oscillating heat pipe," in Proceedings of 11th International Heat Pipe Conference, pp. 166-169, Tokyo, Japan, 1999.
[7] Q. Huang, "Experimental study on the startup performance of solar heat pipe," Beijing University of Technology, 2009.

[8] L. Jingtao, L. Zhihong, and H. Zhenxing, "Flow pattern and the flow direction analysis of the pulsating heat pipe," Thermal Power Engineering, vol. 24, no. 3, pp. 347-351, 2009.

[9] L. Xiangdong and H. Yingli, "The oscillating heat pipe in closed cycle two-phase flow numerical simulation," Southeast University, vol. 39, no. 5, pp. 961-965, 2009.

[10] W. Jiansheng, W. Zhenchuan, and L. Meijun, "Thermal performance of pulsating heat pipes with different heating patterns," Applied Thermal Engineering, vol. 64, no. 1-2, pp. 209-212, 2014.

[11] H. Tingting, "The effect of hydrophilic and hydrophobic properties on the transmission properties of pulsating heat pipes," Dalian University of Technology, 2014.

[12] S. Kim, Y. Zhang, and J. Choi, "Effects of fluctuations of heating and cooling section temperatures on performance of a pulsating heat pipe," Applied Thermal Engineering, vol. 58, no. 1-2, pp. 42-51, 2013.

[13] T. Hao, X. Ma, Z. Lan, and Y. Zheng, "Experimental study on liquid slug liquid film deposition in superhydrophilic pulsating heat pipe," Journal of Engineering Thermophysics, vol. 1, pp. 168-171, 2015.

[14] Y. Zhang, A. Faghri, and M. B. Shafii, "Analysis of liquidvapor pulsating flow in a U-shaped miniature tube," International Journal of Heat and Mass Transfer, vol. 45, no. 12, pp. 2501-2508, 2002.

[15] AIAA, "Oscillatory flow in pulsating heat pipes with arbitrary number of turns," Journal of Thermophysics \& Heat Transfer, vol. 17, no. 3, pp. 340-347, 2003.

[16] X. Zhang, Z. Ren, and F. Mei, Heat transfer theory, vol. 75, China Architecture and Building Press, Beijing, 2007.

[17] Q. Yueping, S. Huaitao, W. Jiansong, and D. Zhanyuan, "Analysis of the temperature of the surrounding rock periodic boundary finite volume method," Coal Society, vol. 40, no. 7, pp. 1541-1549, 2015.

[18] S. Yang and W. Tao, Heat Transfer, Higher Education Press, 2011. 

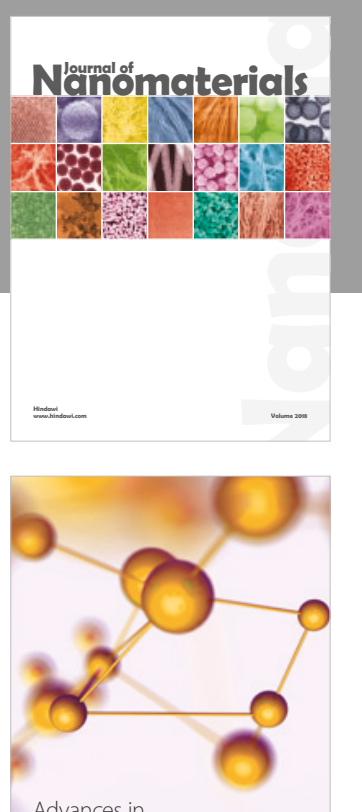

Physical Chemistry
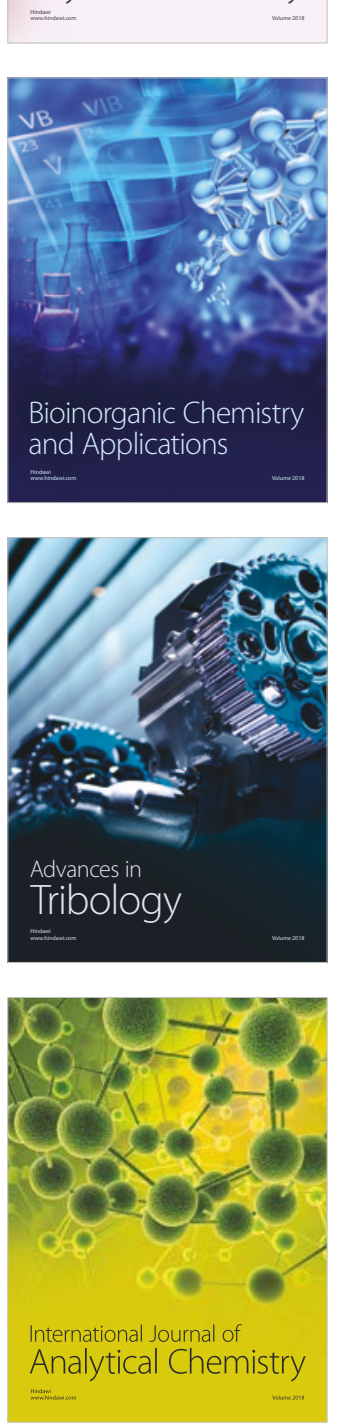

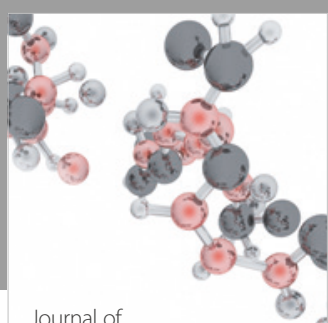

Analytical Methods

in Chemistry

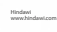

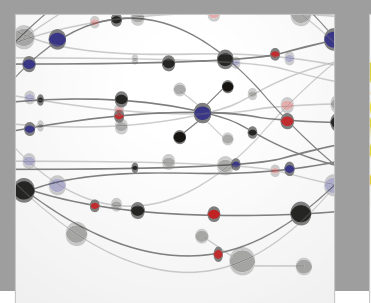

The Scientific World Journal

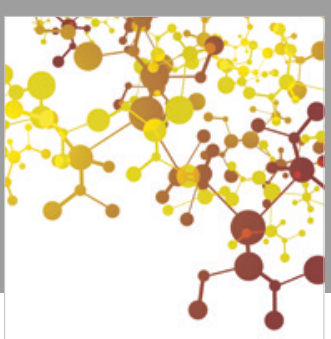

Journal of

Applied Chemistry
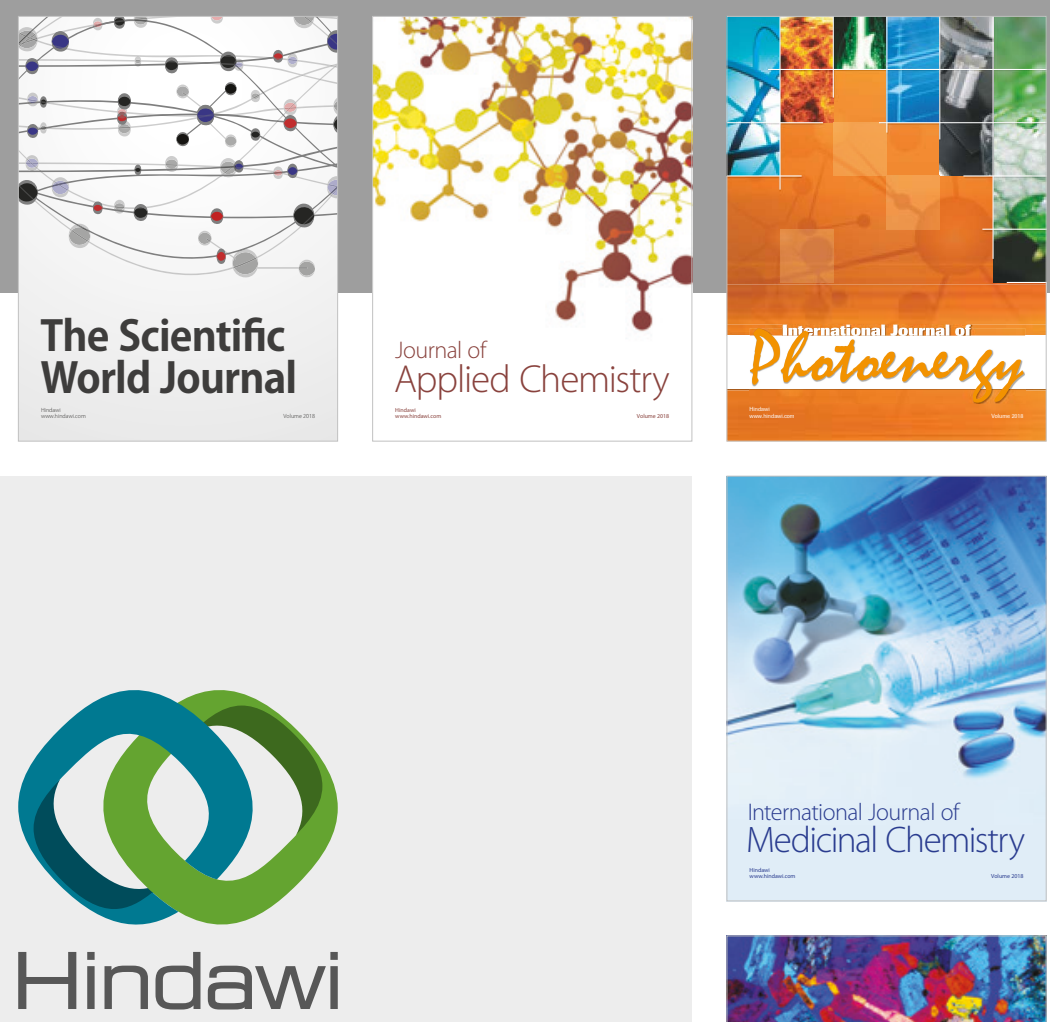

Submit your manuscripts at

www.hindawi.com
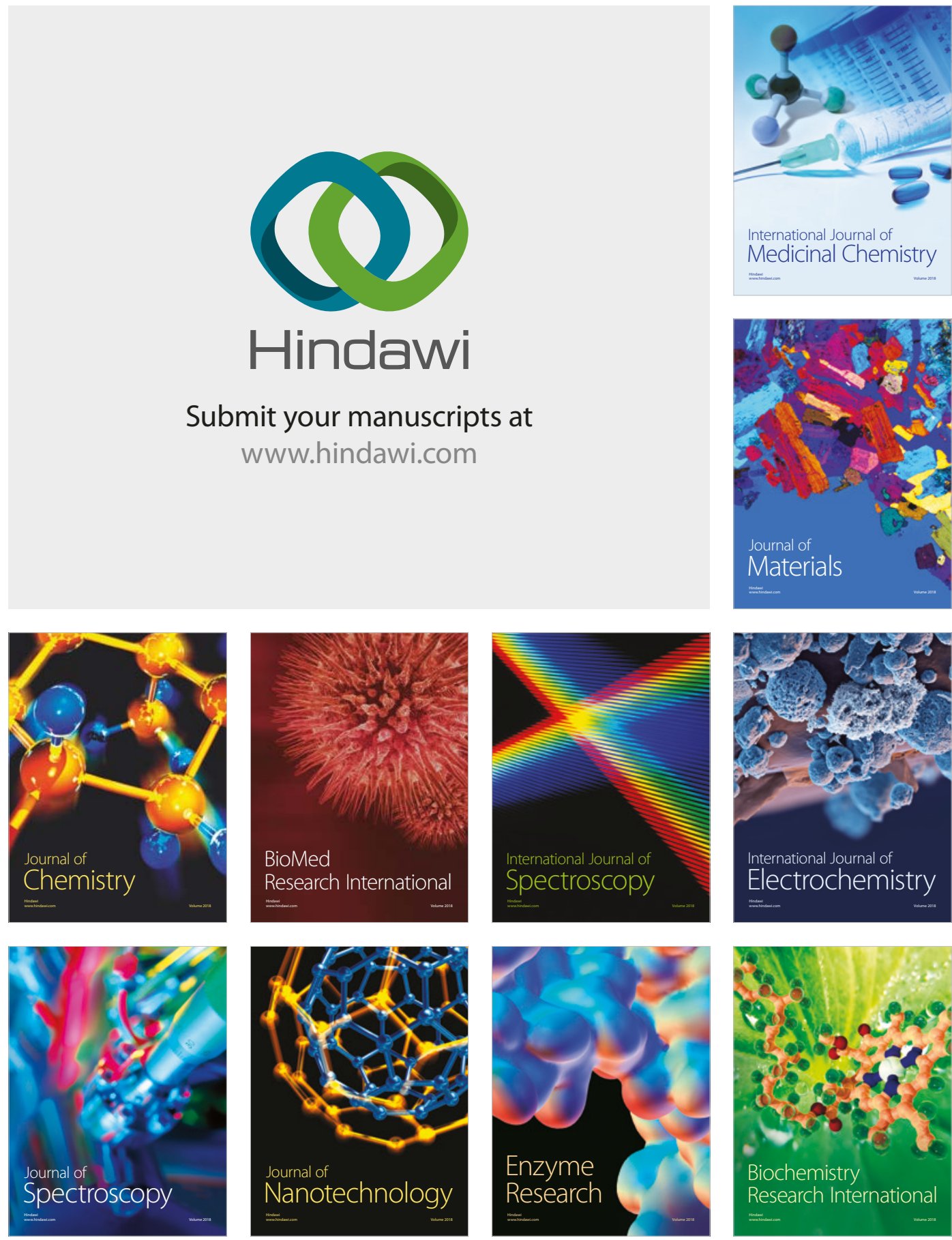
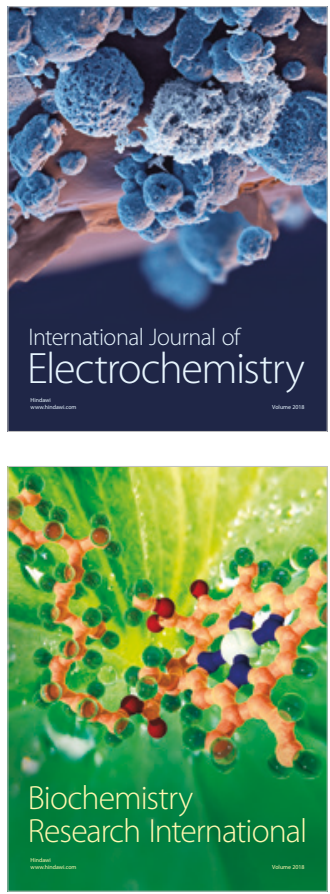